University of Wollongong

Research Online

Faculty of Engineering and Information

Faculty of Engineering and Information

Sciences - Papers: Part B

Sciences

2018

Device-to-Device Communication in 5G: Towards Efficient Scheduling

Jana Fayek

University of Wollongong

Mohamad Aoude

Lebanese International University

Mohamad Raad

Lebanese International University

Raad Raad

University of Wollongong, raad@uow.edu.au

Follow this and additional works at: https://ro.uow.edu.au/eispapers1

Part of the Engineering Commons, and the Science and Technology Studies Commons

Research Online is the open access institutional repository for the University of Wollongong. For further information contact the UOW Library: research-pubs@uow.edu.au 


\title{
Device-to-Device Communication in 5G: Towards Efficient Scheduling
}

\begin{abstract}
$5 \mathrm{G}$ wireless networks are expected to carry large traffic volumes due to the growth of mobile devices and the increasing demand for high data rates from applications. Device to device communication is one of the suggested technologies to support this increasing load and enhance the capacity of networks. However, the implementation of D2D communication reveals many barriers that include communication scheduling, for which the architecture remains complex and obscure. In this paper, an overview of the available literature on the implementation of networks supporting D2D communication is presented, emphasizing the complexity of the offered solutions. This paper also offers a study of the impact of different device distribution models on the throughput of the devices. The paper introduces the challenges and makes the case for the need to find a more efficient D2D scheduler providing less complexity.
\end{abstract}

\section{Keywords}

efficient, scheduling, towards, device-to-device, $5 \mathrm{~g}$ :, communication

Disciplines

Engineering | Science and Technology Studies

\section{Publication Details}

J. Fayek, M. Aoude, M. Raad \& R. Raad, "Device-to-Device Communication in 5G: Towards Efficient Scheduling," International Journal of Digital Information and Wireless Communications, vol. 8, (3) pp. 144-149, 2018. 


\title{
Device-to-Device Communication in 5G: Towards Efficient Scheduling
}

\author{
Jana Fayek, Mohamad Aoude, Mohamad Raad, Raad Raad \\ jaf462@uowmail.edu.au, University of Wollongong, Australia, \\ maoude@ul.edu.lb, Lebanese University, Lebanon \\ Mohamad.raad@liu.edu.lb, Lebanese International University, Lebanon \\ raad@uow.edu.au, University of Wollongong, Australia
}

\begin{abstract}
G wireless networks are expected to carry large traffic volumes due to the growth of mobile devices and the increasing demand for high data rates from applications. Device to device communication is one of the suggested technologies to support this increasing load and enhance the capacity of networks. However, the implementation of D2D communication reveals many barriers that include communication scheduling, for which the architecture remains complex and obscure. In this paper, an overview of the available literature on the implementation of networks supporting D2D communication is presented, emphasizing the complexity of the offered solutions. This paper also offers a study of the impact of different device distribution models on the throughput of the devices. The paper introduces the challenges and makes the case for the need to find a more efficient D2D scheduler providing less complexity.
\end{abstract}

\section{INTRODUCTION}

Smart devices are becoming ubiquitous; these devices are supported by all-IP fourth generation LTE networks. The global mobile traffic encountered a growth of around 70\% [1] in 2014 , where $26 \%$ of the total global mobile devices were smartphones and were responsible for $88 \%$ of the total mobile data traffic. This was the result of the user-oriented mobile multimedia applications such as mobile video conferencing and video streaming. Cisco's Visual Networking Index (VNI) forecasted that smart devices will compose over half of the connected devices to mobile networks by 2019. With this expected increase of smartphone usage and its consequential data traffic, new technologies are needed to address this issue and work on enhancing the network's capacity in terms of supporting a larger number of users, noting that $5 \mathrm{G}$ wireless systems are expected to support these technologies while improving data rates and quality of service. Some of the related work in this context include the Internet of Vehicles (IoV), Device to Device communications and Machine to Machine (M2M) communications.

$5 \mathrm{G}$ wireless communications envision [1] a significant increase of wireless data rates, bandwidth, coverage and connectivity with a decrease of latency and energy consumption. Considering the network capacity, the traffic volume in $5 \mathrm{G}$ networks is predicted to attain tens of Exabytes ( $2^{60}$ Bytes) on a monthly basis [2], which may require new approaches for the network design and operation to boost the capacity of the future networks. Such approaches include the intervention of users in storage, relaying and content delivery operations, moving gradually from Base Station (BS) centric networks into device centric networks. In this context, device to device (D2D) communication was first introduced in the 3GPP LTE-A system as a promising solution to enhance spectral efficiency and increase the system capacity [3].

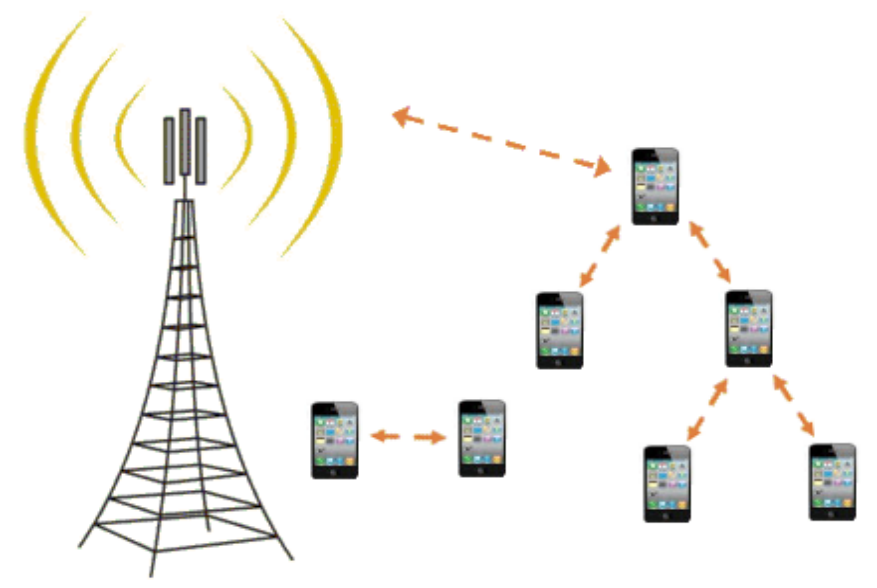

Fig. 1. Device-to-device communications in cellular networks

Identified as the direct communication between two mobile users without passing through the base station [3], [4], D2D communication is more likely to be applied in today's cellular networks where mobile users are potentially in high physical proximity and in range for direct communication as shown in Fig. 1. Without the relay of the BS, the D2D devices will communicate with each other over a direct link using the cellular spectrum while remaining under the control of the BS for the administrative functions such as resource allocation and power control allowing resource planning and scheduling. Using D2D communication, the D2D mobile users will share the resources of the cellular users which improves the bandwidth utilization and the spectral efficiency. By sharing the same resources among users and bypassing the BS, the traffic volume is reduced and the capacity of networks is increased, which allows networks to support a larger number of users answering the expected largely increasing traffic 
volumes in future networks. However, the interference between cellular and D2D links appears to be the main issue of D2D communication, for which many scheduling models were already presented in the literature, exposing a high level of complexity and subsequently several difficulties in the employment in realistic networks [3]. As shown in Fig. 2, a scheduling algorithm mainly constitutes a resource allocation phase which distributes resources among cellular and D2D users according to specific rules and inputs which differ from a scheduler to another. The next step is the spectrum sensing where the network requirements are evaluated to check whether they are achieved or not, consequently determining the network's state. These scheduling steps may reveal a considerable level of complexity which varies between distinct algorithms.

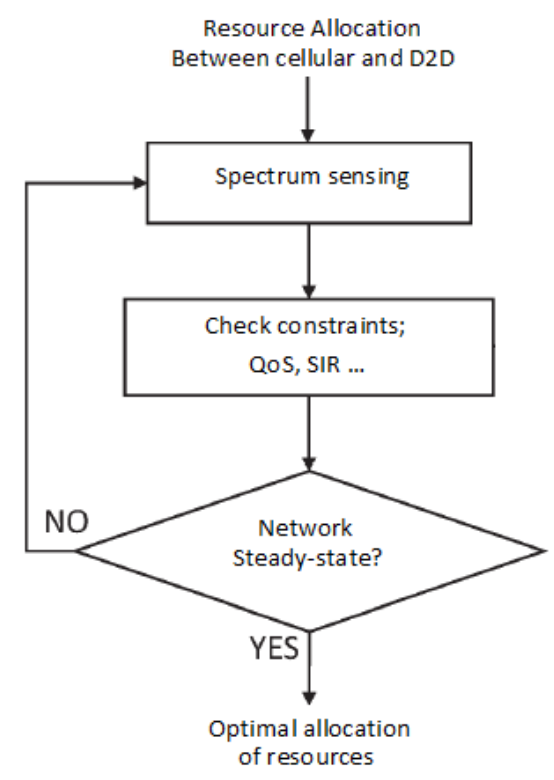

Fig. 2. Flow diagram of a scheduling algorithm.

In this paper, the complexity issue of the available schedulers is discussed in section II, along with two examples of existing schedulers presenting algorithms to address the interference issue of D2D communication. Section III presents a literature review of the related works in the same context of D2D scheduling including a comparison between the schedulers and reflecting their complexity. Section IV presents a non-uniform distribution model and a uniform model, comparing their average D2D throughput results, emphasizing on the importance of using a realistic distribution model. The paper is finally concluded in section $\mathrm{V}$.

\section{STATE-OF-THE-ART RESEARCH WORKS}

Device to device communication was originally presented in [5] to enable multihop relays in cellular networks. The first attempted implementation for D2D communication in a cellular network was built by Qualcomm's FlashLinQ [6] in 2010, followed by many other projects adopting the different types of D2D communications. FlashLinQ achieved high data rates at long communication ranges over licensed spectrum, however, it caused inefficiency in resource reuse because link scheduling was achieved exclusively by its transmitter and receiver nodes [7].

Known as Inband D2D, and detailed in [3-4], D2D and cellular links share the same spectrum, provoking high complexity with the resource distribution and interference between transmissions. In order to manage the interference between transmissions, many scheduling techniques were proposed based on resource allocation schemes where resources are distributed on the users following opportunistic criteria or specific measures and rules [7-13].

The proposed schedulers differ algorithmically, however, all impose significant complexity, whether in their consistent requirement for data inputs from the users, such as the channel state, or because of the mathematical operations included in the algorithms; many algorithms consisted of significant series of computations in order to allocate the resources based on these estimations' results, which subsequently reflected a computational complexity that becomes more considerable for large networks. On the other hand, many researchers suggested opportunistic resource scheduling algorithms that allocate resources based on a pre-defined parameter, such as the system state and the average data rate of links, which signifies more traffic in the network and less simplicity in the technique.

The available D2D schedulers lack the simplicity required for practical implementation. Our aim is to develop a straight forward efficient scheduler minimizing the main issue of interference between the communications and optimizing the efficiency of deploying D2D communication. Further in this section, two examples of complex schedulers are described in detail.

To start with, a scheduler is a pre-prepared algorithm that handles a specific schedule of tasks and operations, which in the case of D2D communication, aims for minimizing the interference between $\mathrm{D} 2 \mathrm{D}$ and cellular transmissions by allocating appropriate channel time (i.e. resources). It may include numerical simulations, rules and conditions, and distributive decisions of resources. The two example schedulers discussed next present a channel-aware link scheduling algorithm which requires the channel states of the links for its resource distribution algorithm, and an opportunistic scheduler that offers the option of $\mathrm{D} 2 \mathrm{D}$ relaying.

\section{A. Channel-aware distributed link scheduling [8]}

For this D2D scheduler, the authors consider an OFDM system of several D2D links where each traffic slot is regarded 
as a time-slot; each D2D link $\mathrm{K}$ involves a transmitter $u_{K}^{T}$ and a receiver $u_{K}^{R}$ where $\mathrm{K}=\{1,2, \ldots, \mathrm{K}\}, U^{T}$ and $U^{R}$ denote the set of links, the set of transmitters and the set of receivers respectively. The system assumes a time-varying wireless channel that remains unchanged during a traffic slot. The set of system states is designated as $\mathrm{S}=\{1,2, \ldots, \mathrm{S}\}$ where the chance of each system state $\mathrm{s}$ in a traffic slot is denoted as $\pi_{S}$.

To represent link scheduling, the authors in [8] identify a scheduling group $\mathrm{z}$ as a subset of $\mathrm{K}$ and a scheduling indicator $q_{Z}^{S} \in\{0,1\}$ where 1 means that the links in the scheduling group $\mathrm{z}$ are scheduled in a traffic slot with system state $\mathrm{s}$ and 0 signifies otherwise. Next, the scheduling vector indicating which links are scheduled in a traffic slot is defined and denoted by $Q_{S}$.

Supposing that each transmitter has data to transmit to its receiver, the TXs (transmitters) of the scheduled links transmit their data with fixed transmission power, $P_{k}$ over a link k, and using the entire wireless channel. Using the Shannon capacity formula, the achievable instantaneous data rate of a link $\mathrm{K}$ is derived and represented as $r_{k}^{S}\left(\overline{q_{s}}\right)$ and then the average data rate of link $\mathrm{k}$ is calculated as $\sum_{s \in S} \pi_{S} r_{k}^{S}\left(\overline{q_{s}}\right)$ and the total average sum-rate of the system would be obtained as $\sum_{Z \in Z} \sum_{s \in S} \pi_{s} r_{k}^{S}\left(\overline{q_{s}}\right)$, noting that the average data rate of link $\mathrm{k}$ has a minimum average rate requirement $\xi_{k}$

In [7], the link scheduling problem is expressed as maximizing the total average sum-rate of the system while maintaining a minimal required data rate of every link as

$$
\begin{aligned}
& \sum_{z \in Z} \sum_{s \in S} \pi_{s} r_{k}^{s}\left(\overline{q_{s}}\right) \geq \xi_{k}, \\
& \forall k \in K, \forall \overline{q_{s}} \in Q_{s}, \forall s \in S
\end{aligned}
$$

In the developed algorithm, the scheduling indicator is evaluated by the central controller and represented in a function of $\bar{\lambda}^{(t)}$ where $\bar{\lambda}^{(t)}$ stands for the Lagrangian multiplier vector of the dual problem in (2), which is represented after the transmission as a function of $\alpha^{(t)}$, a step size at time-slot $\mathrm{t}$, and $v_{k}^{(t)}$, the stochastic subgradient of the dual problem in (1) which can be obtained by Danskin's Theorem [3] as

$$
v_{k}^{(t)}=r_{k}^{s}(t)\left(\bar{\lambda}^{(t)}\right)-\xi_{k}, \forall k \in K
$$

Where $r_{k}^{S}{ }^{(t)}\left(\bar{\lambda}^{(t)}\right)$ is the achieved instantaneous data rate of link k for certain $s^{(t)}$ and $\bar{\lambda}^{(t)}$. The Lagrangian multiplier vector in (2) approaches the best solution for $\bar{\lambda}^{*}$ with possibility 1 as the time-slot t tends to infinity if the step size $\alpha^{(t)}$ meets the conditions of $\alpha^{(t)} \geq 0, \sum_{t=0}^{\infty} \alpha^{(t)}=$ $\infty$ and $\sum_{t=0}^{\infty}\left(\alpha^{(t)}\right)^{2}<\infty$

In which case, the scheduling indicator $\bar{q}\left(\bar{\lambda}^{*}\right)$ is the optimal solution of the problem (1).

To perform this optimal algorithm, all links' channel states are communicated with the central controller, which requires considerable signaling overhead. And as the amount of scheduling groups raises with the links' number, the computational complexity of the proposed solution increases. Consequently, the optimal algorithm becomes difficult for practical implementation as a result of its significant signaling overhead and high computational complexity.

\section{B. Opportunistic scheduling with D2D relaying [9]}

Supposing that the cellular network can utilize UEs as D2D relaying UEs, the authors in [9] presented a model which considers a single cell in an OFDMA cellular system consisting of one BS and M UEs that include $\mathrm{K}$ D2D relaying $\mathrm{UE}$, so $\mathrm{K}$ is a subset of $\mathrm{M}$. Based on a static resource allocation, the first $\mathrm{K}$ UEs among $M$ are set to be D2D relaying UEs and the base station is given the index 0 . If D2D relaying UEs function in a half-duplex mode, these UEs would not be receiving and transmitting data simultaneously during one time-slot. In this study, a node stands for either a BS or a UE, and the link from a transmitter node $i$ to a receiver node $j$ over which the data is intended to the destination node $k$ would be denoted by $(i, j, k)$. The model in [9] assumes a time-varying channel that remains intact during a time-slot, denoting the set of subchannels by $\mathrm{N}$ and the set of system states by S.

At the beginning of each times-lot, the BS assigns each subchannel to only one link, defining the subchannel allocation indicators as $q_{(\mathrm{i}, \mathrm{j}, \mathrm{k})}^{n, S}$ which equals to 1 when a subchannel $n$ is assigned to the link, and is null otherwise.

The authors in [9] use the Shannon capacity formula to get the achievable instantaneous data rate on the subchannel $n$ through the link $(i, j, k)$ in a time-slot with system state $\mathrm{s}$ as

$$
\mathrm{r}_{(\mathrm{i}, \mathrm{j}, \mathrm{k})}^{n, S}=\frac{W}{N} \log _{2}\left(1+a_{(\mathrm{i}, \mathrm{j}, \mathrm{k})}^{n, S} \mathrm{p}_{i}\right)
$$

Where $\mathrm{W}$ is the total frequency bandwidth and $a_{(\mathrm{i}, \mathrm{j}, \mathrm{k})}^{n, S}$ is the SINR of link $(\mathrm{i}, \mathrm{j}, \mathrm{k})$, and its total instantaneous data rate would be calculated as

$$
R_{(\mathrm{i}, \mathrm{j}, \mathrm{k})}^{n, S}=\sum_{n \in N} q_{(\mathrm{i}, \mathrm{j}, \mathrm{k})}^{n, S} \mathrm{r}_{(\mathrm{i}, \mathrm{j}, \mathrm{k})}^{n, S}
$$

From this definition, the average data rate on link (i,j,k) would be $\sum_{S \in S} \pi_{S} \mathrm{R}_{(\mathrm{i}, \mathrm{j}, \mathrm{k})}^{n, S}$ where $\pi_{S}$ is the probability of a system state $s$ in a time-slot. From this equation, each UE's the average data rate is generated as the average sum-rate between the BS and a UE k directly, and that between the BS and other $\mathrm{D} 2 \mathrm{D}$ relaying UEs having a UE $\mathrm{k}$ as their destination, while noting that this average rate must guarantee a required minimum data rate $p_{k}$ as

$$
\sum_{S \in S} \pi_{S}\left(\mathrm{R}_{(0, \mathrm{k}, \mathrm{k})}^{n, S}+\sum_{j \in K \backslash(K)} \mathrm{R}_{(0, \mathrm{j}, \mathrm{k})}^{S}\right) \geq p_{k},
$$

\section{Where $K \in M$.}

Assuming that each D2D relaying UE has a maximum average sum-rate for relaying which is predefined as $U_{i}$, the condition would be shown as

$$
\sum_{s \in S} \pi_{s} \sum_{j \in M \backslash(i)} \mathrm{R}_{(i, \mathrm{j}, \mathrm{j})}^{S} \leq U_{i}
$$

Where $i \in K$. 
International Journal of Digital Information and Wireless Communications (IJDIWC) 8(3): 144-149

The Society of Digital Information and Wireless Communications, 2018 ISSN: 2225-658X (Online); ISSN 2412-6551 (Print)

For this system model, the authors formulated an optimization problem and later presented a scheduler aiming at solving it, which has, in their own study, a high computational complexity.

\section{RELATED WORKS}

In addition of the two examples previously detailed, a summary of some schedulers is stated as follows, noting that all the included models showed certain complexity either in their mathematical calculations, or in their network adaptation, or in their requirement for consistent information from the users, detailed in their performance analysis.

A resource allocation algorithm using a time division scheduler is proposed in [10], where the base station's period is partitioned into timeslots where D2D users are evenly given various timeslots for communication supporting more D2D candidates in the system taking into consideration a minimal data rate requirement of every $\mathrm{D} 2 \mathrm{D}$ pair. The authors in [11] present a D2D scheduling technique designed based on the Cellular Fairness Scheduling (CDF) framework which requires the channel state information CSI from the users. In [12], a distributed scheduling approach was proposed for overlaying cellular networks where every D2D link senses the local interference and self-adapts its individual time-frequency (TF) allocation. In [7], the authors proposed two link scheduling methods using a binary matrix that indicates the interference between links, where each active link transmits a Direct Power Signal DPS so the receivers measure the received signal power, and the selection of a direct communication is based on the Signal-to-Interference Ratio SIR estimated values. In [13], a solar energy harvesting based model was proposed to maximize the throughput of an overlay in-band D2D network.

These schedulers are listed in Table.1; the listed schedulers either require input data from the users or need numerical simulations in their algorithms. These constraints increase the complexity of a scheduler and indicate overhead to the network in the case of input requirements.

Considering the complexity of the existing D2D schedulers, further research projects can be directed toward finding new solutions with straightforward algorithms, reducing overhead to the network and minimizing the complication of mathematical simulations. This issue can be extended to study the possibility of developing a scheduler that requires the least information from the users, while depending on more factors that can be estimated by the core network. On the other hand, simpler schedulers are required to include the fewest uncomplicated numerical simulations as much as possible to reduce the computational complexity. Creating a simple yet efficient scheduler is a challenge in D2D communication, which remains open for further assessment as a possible research direction.

More specifically we propose using device centric schedulers such as the one presented in [16]. In this case, each pair of nodes that are within range set up a pseudo random channel access sequence. Scheduling Algorithms presented in [14] and [15] can also be applied to the D2D problem at hand.
TABLE I. D2D SCHEDULERS IN THE LITERATURE

\begin{tabular}{|l|c|c|}
\hline Scheduler & Data Required & Complexity \\
\hline $\begin{array}{l}\text { Channel-aware link } \\
\text { scheduling [7] }\end{array}$ & $\begin{array}{c}\text { Channel State } \\
\text { Information CSI }\end{array}$ & High \\
\hline $\begin{array}{l}\text { Opportunistic } \\
\text { scheduling with } \\
\text { D2D relaying [8] }\end{array}$ & X & Medium \\
\hline $\begin{array}{l}\text { Time division } \\
\text { scheduling [9] }\end{array}$ & $\begin{array}{c}\text { Channel State } \\
\text { Information CSI }\end{array}$ & Low \\
\hline $\begin{array}{l}\text { Cellular Fairness } \\
\text { scheduling [10] }\end{array}$ & $\begin{array}{c}\text { Channel State } \\
\text { Information CSI }\end{array}$ & Low \\
\hline $\begin{array}{l}\text { Distributed } \\
\text { interference } \\
\text { coordination [11] }\end{array}$ & $\begin{array}{c}\text { Quality of } \\
\text { Service QoS }\end{array}$ & High \\
\hline $\begin{array}{l}\text { On-off interference } \\
\text { map scheduling } \\
\text { [12] }\end{array}$ & $\begin{array}{c}\text { Signal to } \\
\text { interference ratio } \\
\text { SIR }\end{array}$ & Low \\
\hline
\end{tabular}

Moreover, the assumed distribution model has a significant impact on the performance of the network, so the scheduler needs to take a realistic distribution into account, noting that most of the current works use uniform distribution models.

In the following section, we present a realistic non-uniform distribution model and a uniform one, to show their performance differences, and their consequent effect on interference scheduling.

\section{D2D DISTRIBUTION}

In most available works, the users' distribution within a cell is usually assumed uniform [17]. However, in a realistic cellular network, the users are usually distributed nonuniformly creating hot spots with high density of users requesting access to the network and other spots with less density of users. In this section, we propose a user distribution model to graph a non-uniform distribution and then calculate the average D2D throughput in the network while varying the number of users. Another random distribution model representing a uniform distribution is later included to compare the throughput results and assess both distribution types.

To model the user probability density function for a nonuniform distribution, we use a two-dimensional 2-D truncated Gaussian function [17] where the cell center is the origin of the coordinates. For a cell of radius 500 m, Fig. 3 represents a symmetrical 2-D truncated Gaussian user distribution over the cell region.

For the non-uniform distribution model shown in Fig. 3 , a radius parameter is added for $\mathrm{D} 2 \mathrm{D}$ communication, where an algorithm splits the users between cellular and D2D based on their geographical proximity. Setting the D2D radius to be $20 \mathrm{~m}$, the distribution of users between cellular and D2D is shown in Fig. 4. 


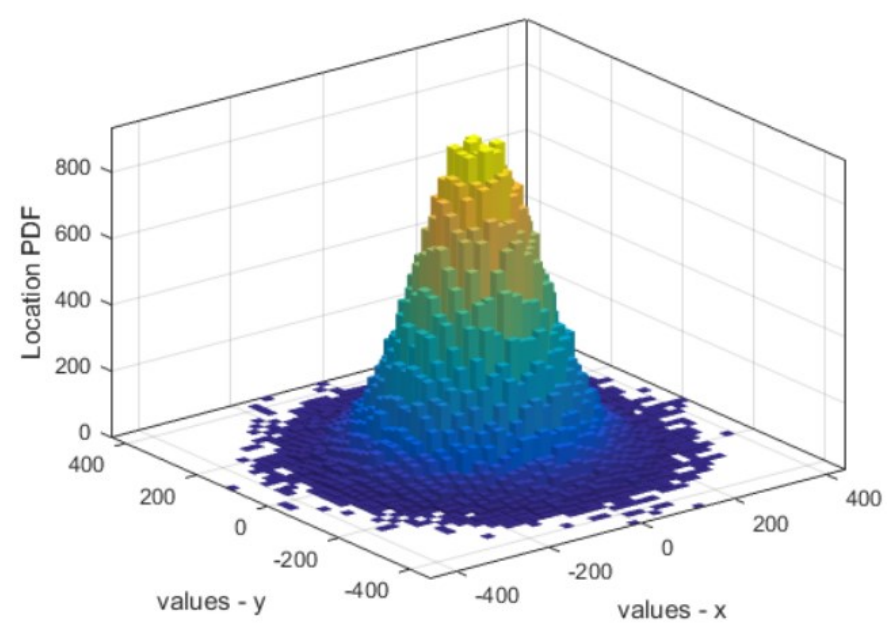

Fig. 3. Symmetrical 2-D truncated Gaussian user distribution pdf over a cell region

As shown in Fig. 4, the number of users in range for direct communication is significant which increases the probabilities of using D2D communication. Next we will calculate the average D2D throughput in the network below.

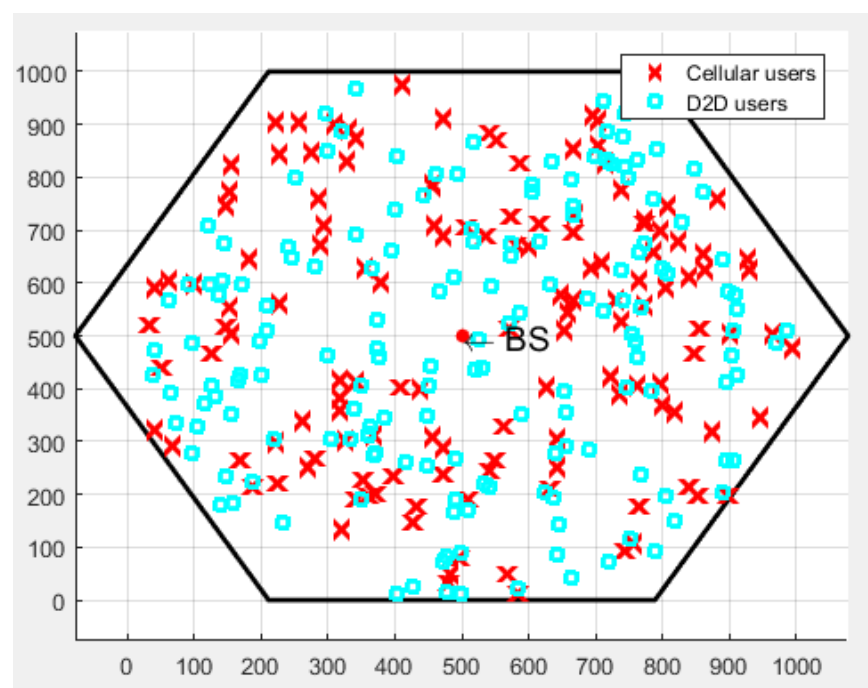

Fig. 4. D2D and Cellular users' locations map using the non-uniform Gaussian distribution

For the above distribution, the device to device communication consists of D2D pairs only where each D2D user is suggested to be given one resource block; for each $\mathrm{D} 2 \mathrm{D}$ receiver, the received power from the $\mathrm{D} 2 \mathrm{D}$ transmitter is first calculated. Then, we calculate the power received from all other channels which are considered as interfering channels, and which we call the interference power. From the received interference power, the $\mathrm{D} 2 \mathrm{D}$ signal to interference plus noise ratio SINR is then obtained for each D2D pair in the network.
Next, we calculate the D2D throughput as a sum function in terms of the resulting SINR values, presented as

$$
\sum \log _{2}(1+S I N R)
$$

This process of calculation is performed for each D2D pair in the network as a loop, while summing the D2D throughputs at the end of the loop cycle. Finally, the average D2D throughput is calculated by dividing the total D2D throughput on the number of performed loops.

To assess the results, we use a uniform distribution model and repeat the same process to get its D2D average throughput. To start with, the distribution of users between cellular and D2D using the uniform distribution model is shown in Fig. 5.

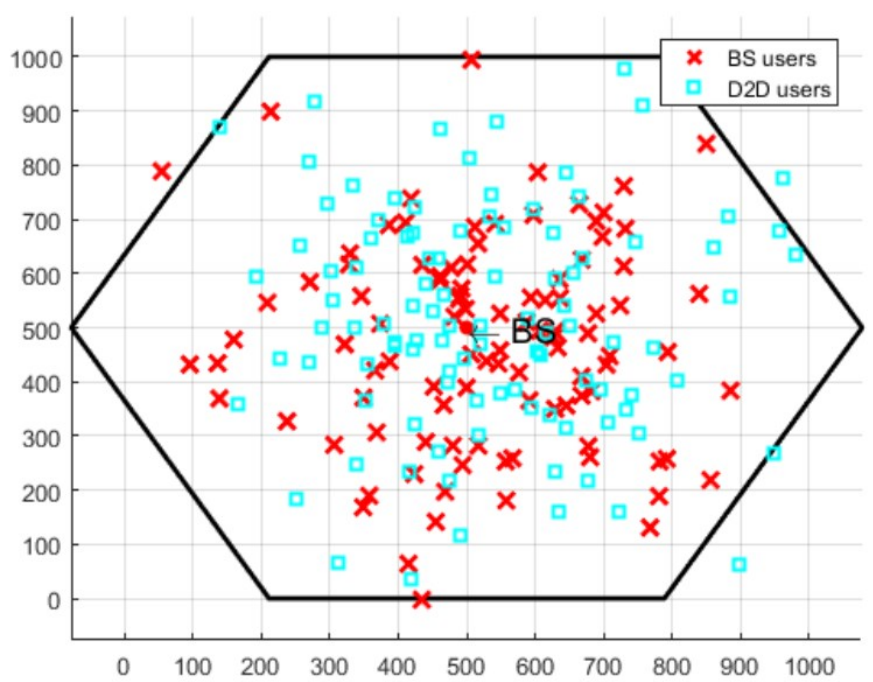

Fig. 5. D2D and Cellular users' locations map using random distribution

For both distribution models, the average D2D throughput is calculated using the process previously detailed, while varying the number of users between 50 and 500 . The results are plotted in Fig. 6, where the average D2D throughput for the Gaussian distribution shows better results than that of the random distribution.

As mentioned before, most available works used static and uniform distribution models, to either reduce the complexity of their systems or to adopt static assumptions, which consequently assumes that their results would probably decrease when applying them on realistic networks. However, our previous simulations showed better simulation results for the average D2D throughput in a Gaussian distribution model, which is a non-uniform realistic distribution model, than the results of a uniform distribution model. These results validates the possibility of working on realistic network scenarios, and by that, studying the optimization of interference scheduling 
algorithms on such scenarios rather than on uniform models, as it is mostly done in previous works.

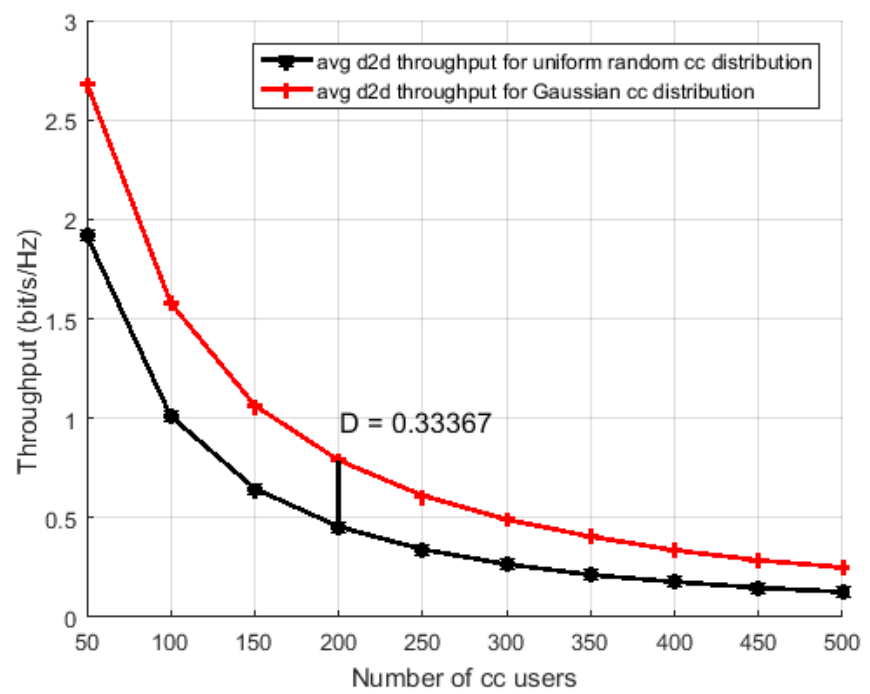

Fig. 6. Average D2D throughput for random and Gaussian distributions vs the number of users

\section{CONCLUSION}

A major challenge for $5 \mathrm{G}$ networks is the massive traffic volume to be expected due to the increasing demand for data. Device to device communication was presented as a promising solution, while regarding its interference issue. Many existing scheduling techniques were listed in this paper, with a detailed summary of two significant schedulers.

The main problem observed in all of the reviewed papers is the complexity of the schedulers and their assumed uniform distribution models. To improve the efficiency of deploying device to device communication, there is a need to direct the search for a less-complex D2D scheduler, providing an easy and efficient implementation in realistic networks, while maintaining the initial D2D communication goals of increasing throughput, reducing overhead and enhancing the spectral efficiency.

\section{REFERENCES}

[1] M. Agiwal, A. Roy and N. Saxena, "Next Generation 5G Wireless Networks: A Comprehensive Survey," IEEE COMMUNICATIONS SURVEYS \& TUTORIALS, vol. 18, pp. 1617-1655, 2016.

[2] S. Buzzi, C.-L. I, T. Klein, V. Poor, C. Yang and A. Zappone, "A Survey of Energy-Efficient Techniques for 5G Networks and Challenges Ahead," IEEE JOURNAL ON SELECTED AREAS IN
COMMUNICATIONS, vol. 34, pp. 697-709, 2016.

[3] A. Asadi, Q. Wang and V. Mancuso, "A Survey on Device-toDevice Communication in Cellular Networks," IEEE COMMUNICATION SURVEYS \& TUTORIALS, vol. 16, pp. 1801-1819, 2014.

[4] J. Liu, N. Kato, J. Ma and N. Kadowaki, "Device-to-Device Communication in LTE-Advanced Networks: A Survey," IEEE COMMUNICATION SURVEYS \& TUTORIALS, vol. 17, pp. 1923-1940, 2015

[5] Y. Lin and Y.-C. Hsu, "Multihop cellular: A new architecture for wireless communications," IEEE INFOCOM, vol. 3, pp. 1273-1282, 2000 .

[6] X. Wu, "FlashLinQ: A sunchronous distributed scheduler for peerto-peer ad hoc networks," Proc. Allerton Conf. Commun., pp. 514521, 2010.

[7] J.-W. Kang, A. Hussain and S.-H. Kim, "Link Scheduling schemes with on-off interference map for device-to-device communications," IET Communications, vol. 9, pp. 359-366, 2015.

[8] J.-W. L. Hyun-Suk Lee, "QoS and Channel-Aware Distributed Link Scheduling for D2D Communication," IEEE, pp. 8565-8579, 2016.

[9] H.-T. R. J.-W. L. Jee Hun Song, "Opportunistic scheduling and incentive mechanism for OFDMA networks with D2D relaying," ELSEVIER COMPUTER NETWORKS, pp. 772-787, 25 September 2015.

[10] J. Z. Y. Z. Biwei Chen, "A Time Division Scheduling Resource Allocation Algorithm for D2D Communication in Cellular Networks," IEEE ICC, pp. 5422-5428, 2015.

[11] B. D. R. P. Nguyen, "Fair Scheduling Policies Exploiting Multiuser Diversity in Cellular Systems with Device-to-Device Communications," IEEE Transactions on Wireless Communications, vol. 14, pp. 4757-4771, 2015.

[12] M. A. Alvarez, G. Soatti and U. Spagnolini, "Device-to-Device Resource Scheduling by Distributed Interference Coordination," IEEE COMMUNICATIONS WORKSHOPS, pp. 505-510, 2016.

[13] U. Saleem, H. K. Qureshi, S. Jangsher and M. Saleem, "Transmission Power Management for Throughput Maximization in Harvesting Enabled D2D Network," IEEE ISCC, pp. 1078 - 1083, 2016.

[14] H. Wang, KW Chin, S. Soh, R. Raad, "A distributed maximal link scheduler for multi Tx/Rx Wireless Mesh Networks”, IEEE Transactions on Wireless Communications, Vol.14, 1,pp 520-531, 2015.

[15] H. Wang, KW Chin, S. Soh, R. Raad, "Novel joint routing and scheduling algorithms for minimizing end-to-end delays in multi Tx-Rx wireless mesh networks", Computer Communications Elsevier, pp. 63-77, 2015.

[16] KW. Chin and R. Raad, "ArDeZ: a low power asymmetric rendezvous MAC for sensor networks", IEEE Conference on Computer Communications and Networks, ICCCN 2005, Proceedings 14th International Conference on, pp 99-104, 2005.

[17] X. Tang and H. Yang, "Effect of User Distribution on the Capacity of Cellular Networks," Atlantis Press, National Conference on Information Technology and Computer Science,pp. 370-373, 2012 